\title{
Short vs. long length of rectus femoris during eccentric exercise in relation to muscle damage in healthy males
}

\author{
Vassilis Paschalis ${ }^{\mathrm{a}, *}$, Yiannis Koutedakis ${ }^{\mathrm{a}, \mathrm{b}}$, Vassilios Baltzopoulos ${ }^{\mathrm{c}}$, Vassilis Mougios ${ }^{\mathrm{d}}$, \\ Athanasios Z. Jamurtas ${ }^{a}$, Giannis Giakas ${ }^{\text {a }}$ \\ a Department of Physical Education and Sports Sciences, Thessaly University, Karies 42100, Trikala, Greece \\ ${ }^{\mathrm{b}}$ School of Sport, Performing Arts and Leisure, Wolverhampton University, Walshall, UK \\ ${ }^{\mathrm{c}}$ Center of Biophysical and Clinical Research into Human Movement, Manchester Metropolitan University, Alsager, UK \\ ${ }^{\mathrm{d}}$ Department of Physical Education and Sports Sciences, Aristotle University, Thessaloniki, Greece
}

Received 14 September 2004; accepted 24 February 2005

\begin{abstract}
Background. We investigated the effects of short vs. long length of rectus femoris during eccentric exercise of similar range of motion on selected muscle damage indicators.

Methods. Using an isokinetic dynamometer at $1.05 \mathrm{rad} / \mathrm{s}, 12$ healthy male [mean (standard deviation), 21 (2) years] volunteers randomly underwent two exercise sessions, one on each leg, 14 days apart. During each session, subjects had to accomplish 12 sets of 10 maximal voluntary efforts in seated and prone positions to achieve short and long length of rectus femoris, respectively. Muscle damage indicators [serum creatine kinase activity, delayed onset muscle soreness, range of motion, eccentric peak torque, concentric peak torque and isometric peak torque] were assessed pre-exercise and 24, 48, 72 as well as $96 \mathrm{~h}$ post-exercise.

Findings. Compared to baseline data, creatine kinase, delayed onset muscle soreness, and range of motion disclosed significant changes at all time points after both exercise sessions $(P<0.05)$. However, these muscle damage indicators demonstrated greater changes following exercise at short compared to long length of rectus femoris $(P<0.05)$. Torque assessments also revealed that nine out of 12 and only two out of 12 measurements decreased significantly following short and long length eccentric exercise of rectus femoris, respectively $(P<0.05)$. Short length eccentric exercise resulted in greater torque declines compared to long length during concentric and isometric evaluations $(P<0.05)$. No such differences were observed when torque changes were evaluated eccentrically.

Interpretation. Short length of rectus femoris eccentric exercise induces greater muscle damage and peak torque declines than the corresponding long length in healthy adults.
\end{abstract}

(C) 2005 Elsevier Ltd. All rights reserved.

Keywords: Creatine kinase; Delayed onset muscle soreness; Range of motion; Isokinetic dynamometry

\section{Introduction}

Although muscle tissue is extremely elastic, microdamage may occur in response to unusual exercise de-

\footnotetext{
* Corresponding author.

E-mail address: vpasxal@pe.uth.gr (V. Paschalis).
}

mands, especially when exercise includes eccentric contractions (Hoppeler, 1986). Such muscle damage, in turn, negatively affects muscle performance (Clarkson and Tremblay, 1988; Nosaka et al., 2002). An animal study indicated that muscle length is an important factor in exercise-induced muscle damage (Hunter and Faulkner, 1997). Animal studies have also revealed that sarcomeres under stretching are more susceptible to injury 
than non-stretched sarcomeres (Macpherson et al., 1997), leading to a greater degree of inhomogeneity and disruption of the weaker ones (Morgan, 1990).

In humans, using different range of motion of the biarticular biceps brachii, investigators found that maximal eccentric exercise at long muscle length (LL) (100-180 degrees elbow flexion) brought about greater muscle damage compared to eccentric exercise at short muscle length (SL) (50-130 degrees elbow flexion) (Jones et al., 1989; Newham et al., 1988; Nosaka and Sakamoto, 2001). There is only one study which addressed length changes of the quadriceps femoris (Child et al., 1998). Using different knee range of motion, these authors found that eccentric exercise at LL (120-40 degrees knee angle) produces greater delayed onset muscle soreness and maximal voluntary contraction decrements compared to SL (160-80 degrees knee angle).

The biarticular rectus femoris is the only muscle of the quadriceps femoris that crosses both the hip and knee joint. Therefore, its contribution to torque output is mainly affected by the hip angle (MacIntyre et al., 1998). A hip angle decrease from 180 to 100 was followed by a concomitant $7-10 \%$ decline in the knee extensors torque (Pavol and Grabiner, 2000). It has also been suggested that the higher torque generation and the higher surface electromyographic (EMG) activity by quadriceps femoris found in a SL compared with a LL position has been attributed to voluntary muscle activation (Babault et al., 2003; Maffiuletti and Lepers, 2003).

To our knowledge, there is no literature on the effects of eccentric exercise at different lengths of rectus femoris. Therefore, the purpose of the present study was to investigate the effects of eccentric exercise with SL and LL of rectus femoris utilizing the full range of motion on selected muscle damage indicators [creatine kinase (CK), delayed onset muscle soreness, range of motion (RoM), eccentric peak torque, concentric peak torque and isometric peak torque at $110^{\circ}$ knee flexion] in healthy males.

\section{Methods}

\subsection{Subjects}

Twelve healthy males [their mean (standard deviation) age, height and mass was: 21 (2) years, 179.5 (10) $\mathrm{cm}$ and $78.6(6) \mathrm{kg}$, respectively] volunteered. Exclusion criteria included: experience of scheduled eccentric exercise training, any form of resistance training for at least six months prior to this investigation and taking of antiinflammatory drugs. Subjects read and signed an informed consent form according to the standards of the Thessaly University Ethics Committee.

\subsection{Procedures}

In two separate sessions, we investigated the effects of eccentric exercise with SL and LL of rectus femoris on selected muscle damage indicators. Volunteers randomly underwent the two exercise sessions, one on each leg, 14 days apart. It has been reported that just eight days are adequate to allow muscle damage indicators such as $\mathrm{CK}$, delayed onset muscle soreness, maximal voluntary contractions (MVCs) to recover to their pre-exercise levels (Smith et al., 1994). In each session, subjects had to accomplish 12 sets of 10 eccentric MVCs which lasted approximately $35 \mathrm{~min}$, including resting intervals. In order to investigate the contribution of the biarticular rectus femoris on muscle damage, we changed the hip angle as follows: seated $\left(90^{\circ}\right.$ hip angle) and prone $\left(180^{\circ}\right.$ hip angle) positions in order to achieve SL and LL of rectus femoris, respectively. Using the default musculoskeletal model of the lower limbs in the Software for Interactive Musculoskeletal Modelling (SIMM, Musculographics, USA) as previously described (Delp and Loan, 1995), it was confirmed that the rectus femoris was about $19 \%$ longer when the hip angle was at $180^{\circ}$ (prone position) compared to $90^{\circ}$ (seated position), while knee remained fully extended.

Prior to each exercise session, subjects performed an 8-min warm-up cycling on a Monark ergometer (Vansbro, Sweden) at $70 \mathrm{rpm}$ and $50 \mathrm{~W}$, followed by $5 \mathrm{~min}$ of stretching exercises. To our knowledge, there is no evidence that the type of warm-up used in the present study causes muscle damage in healthy males.

The two eccentric exercise modes were performed on an isokinetic dynamometer (Cybex Norm, Lumex, Ronkonkoma, NY, USA), also used previously in studies of similar nature (Paddon-Jones and Abernethy, 2001; Prou et al., 1999). The dynamometer was calibrated weekly according to the instructions provided by the manufacturer. For technical details, refer to elsewhere (Koutedakis et al., 1997).

Subjects were coupled to the dynamometer by visually aligning the lateral femoral condyle with the axis of rotation of the dynamometer and attaching the ankle cuff proximal to the lateral malleolous. Restrain straps at the thigh and hips were employed throughout. Each subject's functional range of motion was set electronically between $0^{\circ}$ and $120^{\circ}$ of knee flexion to prevent hyperextension and hyperflexion. Gravitational corrections were performed to account for the effect of limb weight on torque measurements. In line with existing reports (Brown et al., 1999; McHugh and Tetro, 2003), the angular velocity of $1.05 \mathrm{rad} / \mathrm{s}$ has been used for all eccentric and concentric activities. This angular velocity was checked by counting the number of revolutions per minute at $1.05 \mathrm{rad} / \mathrm{s}$. Gravitational corrections, angular velocity, feedback on the eccentric exercise intensity and duration of the exercise was automatically provided 
by the isokinetic dynamometer. A two-minute rest between sets was allowed.

\subsection{Muscle damage indicators}

Muscle damage indicators were assessed pre-exercise and 24, 48, 72 as well as $96 \mathrm{~h}$ post-exercise in line with previously published protocols (Newham et al., 1988; Nosaka et al., 2002). Specifically, blood samples were drawn from an antecubital vein into plain evacuated test tubes at both sessions in order to evaluate the activity of $\mathrm{CK}$. The blood was allowed to clot at room temperature for $30 \mathrm{~min}$ and centrifuged at $1500 \mathrm{~g}$ for $10 \mathrm{~min}$. The serum layer was removed and frozen at $-20^{\circ} \mathrm{C}$ until analysis. CK was determined spectrophotometrically (Milton Roy, Spectronic 401, Ivyland, USA) in duplicate using a commercial available kit (Megalab, Athens, Greece). The normal reference range of $\mathrm{CK}$ activity for men using this method is 45-130 IU.

For the evaluations of delayed onset muscle soreness, each subject determined soreness by palpation of the muscle belly and the distal region of the vastus medialis, vastus lateralis and rectus femoris in a seated position with the muscles relaxed. Perceived soreness was then rated on a scale ranging from 1 (normal) to 10 (very, very sore). This scale has been previously documented by other investigators (Clarkson and Tremblay, 1988; Jamurtas et al., 2000).

For the assessment of RoM, the subjects lied prone on the previously described isokinetic dynamometer and their knee joint was attached to the lever arm. A passive flexion was performed by one of the investigators at a very low angular velocity $(0.35 \mathrm{rad} / \mathrm{s})$ and the position where the subject felt any discomfort was taken to indicate the pain-free RoM.

Torque assessments, in relation to muscle damage, have been mainly based on isometric dynamometry (Newham et al., 1987; Saxton et al., 1995; Sayers and Clarkson, 2001). However, daily human movements involve eccentric, as well as concentric and isometric muscle actions, which normally demonstrate different physiological characteristics. Isometric contractions are mainly characterised by elevated intramuscular pressure or increasing concentrations of metabolites associated with fatigue (Vollestad et al., 1988) while the elastic component and efferent neural activation are the main physiological attributes of eccentric (Komi and Bosco, 1978) and concentric actions (Kay et al., 2000) respectively. Therefore, it would be more appropriate to consider all three types of contraction when assessing changes in muscle performance. Details of the assessment protocol appear elsewhere (Koutedakis et al., 1997). In brief, using the same isokinetic dynamometer described above (Cybex Norm), muscle performance indicators of the knee extensors [i.e., eccentric peak torque (EPT), concentric peak torque (CPT) and isometric peak torque at $110^{\circ}$ (IPT) knee flexion] were assessed at the time points of pre-exercise and $24,48,72$ as well as $96 \mathrm{~h}$ post-exercise. The best of three MVCs was recorded for EPT, CPT, and IPT. A three minute rest between contraction modes (eccentric, concentric, and isometric) was allowed, whereas the order of contraction modes was randomised.

\subsection{Data analysis}

Data were normalised to baseline measurements, with $100 \%$ corresponding to pre-exercise level. The Kolmogorov-Smirnov test of normality revealed that none of the variables studied required logarithmic transformation. However, due to lack of homogeneity in variance, non-parametric analyses were applied for CK as previously suggested (Thomas and Nelson, 1996). A $2 \times 5$ (muscle length $\times$ time) repeated measures ANOVA and pair-wise comparisons through simple main effect analysis were used for the remaining studied parameters. The significance level was set at $P<0.05$.

\section{Results}

\subsection{Short vs. long length of rectus femoris during eccentric exercise}

Subjects exercised at intensities corresponding to $85.2 \%(4.1)$ and $88.6 \%(3.5)(P>0.05)$ of their maximal EPT at SL and LL of rectus femoris, respectively. Peak torque declined by $35 \%$ and $27.3 \%$ (Fig. 1) and occurred at the knee angles of $81.2^{\circ}$ (3.6) and $93.6^{\circ}$ (2.9) during SL and LL rectus femoris exercise, respectively, with no significant differences between the two sessions. Torque levels were not significantly different at $90^{\circ}$ of knee angle between the two exercise modes (Fig. 2).

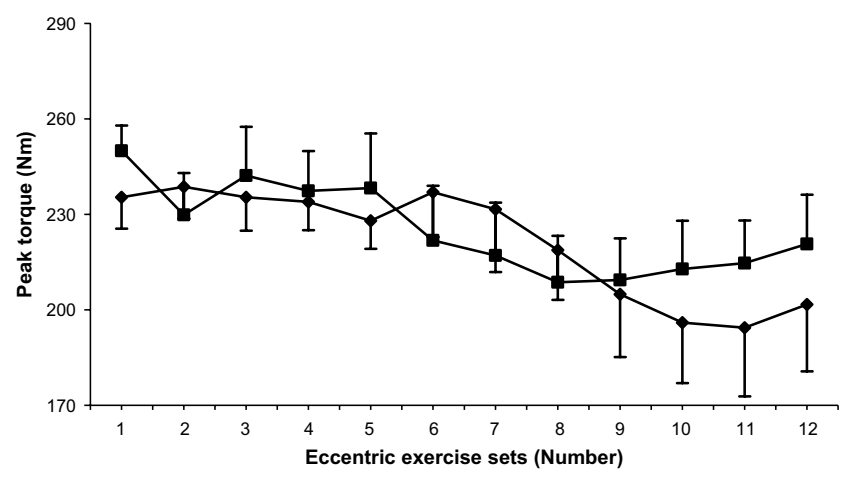

Fig. 1. Changes in eccentric peak torque during eccentric exercise (12 sets) with short ( $)$ and long ( $\boldsymbol{\square}$ ) length of rectus femoris. Error bars denote SEM. 


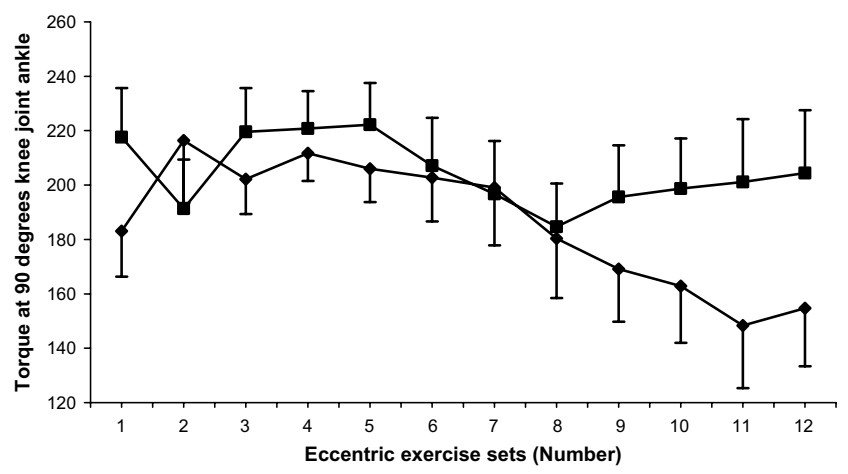

Fig. 2. Changes in eccentric torque during eccentric exercise (12 sets) with short $(\checkmark)$ and long $(\boldsymbol{\square})$ length of rectus femoris at a standardised knee joint ankle $\left(90^{\circ}\right)$. Error bars denote SEM.

\subsection{Muscle damage indicators}

Compared to baseline data, the two eccentric exercise sessions resulted in a significant elevation $(P<0.05)$ of CK activity at all time-points post-exercise (Table 1). However, comparisons between the two sessions revealed that $\mathrm{CK}$ activity was significantly greater only at $72 \mathrm{~h}$ post-SL exercise (Table 1). Also compared to baseline data, delayed onset muscle soreness elevation and RoM reduction were significantly different $(P<0.05)$ even $96 \mathrm{~h}$ post-exercise (Table 1$)$. It is note- worthy that exercise at SL (of rectus femoris) revealed greater $(P<0.05)$ changes in these two muscle damage indicators than the LL equivalent.

Nine out of 12 torque assessments declined significantly over time following eccentric exercise at SL of rectus femoris $(P<0.05$; Table 2$)$. Conversely, only two out of 12 of these assessments declined significantly over time following exercise at LL $(P<0.05)$. Also, exercise at $\mathrm{SL}$ of rectus femoris revealed greater $(P<0.05)$ declines than the LL equivalent in all torque assessment parameters except EPT (Table 2).

\section{Discussion}

We investigated the effects of eccentric exercise at SL and LL of rectus femoris using the same full knee range of motion on selected muscle damage indicators. The two modes of exercise resulted in typical responses (Armstrong, 1990), confirming that muscle damage did occur. The main finding was that eccentric exercise at SL of rectus femoris induces greater muscle damage and causes greater torque declines than the corresponding LL. However, such significant differences were rather surprising given that the rectus femoris contributes only about $17 \%$ of the total isokinetic MVC of knee extension (McNair et al., 1991). These findings could be

Table 1

Percent changes in muscle damage indicators after eccentric exercise performed at short (SL) and long muscle length (LL) of rectus femoris

\begin{tabular}{lllll}
\hline Parameters & $24 \mathrm{~h}(n=12)$ & $48 \mathrm{~h}(n=12)$ & $72 \mathrm{~h}(n=12)$ & $96 \mathrm{~h}(n=12)$ \\
\hline CK-SL (U/L) & $447.8^{*}(81.2)$ & $408.8^{*}(88)$ & $1579.3^{* \#}(435.6)$ & $2216.8^{*}(935.9)$ \\
CK-LL (U/L) & $310.3^{*}(47.4)$ & $298.1^{*}(66.5)$ & $434.2^{*}(157.7)$ & $912.9^{*}(537.9)$ \\
DOMS-SL & $450^{*}(41.7)$ & $650^{*}, \#(43.5)$ & $610^{*}, \#(74.3)$ & $440^{*}, \#(55.7)$ \\
DOMS-LL & $380^{*}(49)$ & $500^{*}(56.4)$ & $420^{*}(49)$ & $310^{*}(51.4)$ \\
RoM-SL (degrees) & $75.9^{*}(4.2)$ & $61.4^{*}, \#(4.8)$ & $61.8^{*, \#}(6.4)$ & $70.6^{*}, \#(5.8)$ \\
RoM-LL (degrees) & $83.0^{*}(2.9)$ & $76.7^{*}(2.7)$ & $80.9^{*}(3.4)$ & $85.8^{*}(3.1)$ \\
\hline
\end{tabular}

CK: creatine kinase; DOMS: delayed onset muscle soreness; RoM: range of motion.

Data are reported as mean (standard error of the mean).

${ }^{*}$ Significantly different $(P<0.05)$ compared to baseline data.

\# Significantly different $(P<0.05)$ between the exercise bouts with SL and LL of rectus femoris.

Table 2

Percent changes in muscle performance indicators after eccentric exercise performed at short (SL) and long muscle length (LL) of rectus femoris

\begin{tabular}{lllll}
\hline Parameters & $24 \mathrm{~h}(n=12)$ & $48 \mathrm{~h}(n=12)$ & $72 \mathrm{~h}(n=12)$ & $96 \mathrm{~h}(n=12)$ \\
\hline EPT-SL $(\mathrm{Nm})$ & $64.9^{*}(5.4)$ & $66.8^{*}(4.9)$ & $72.7^{*}(7.2)$ & $86.5(6.4)$ \\
EPT-LL $(\mathrm{Nm})$ & $65.3^{*}(5.7)$ & $75.5^{*}(5.2)$ & $81.9(6.3)$ & $92.8(7.3)$ \\
CPT-SL $(\mathrm{Nm})$ & $65.7^{*}, \#(4.5)$ & $66.4^{*}, \#(5.5)$ & $73.4^{*}(6.5)$ & $79.4^{*}(4.7)$ \\
CPT-LL $(\mathrm{Nm})$ & $87.7(5.4)$ & $89.8(6.4)$ & $78.0(8.7)$ & $92.8(7.4)$ \\
IPT-SL $(\mathrm{Nm})$ & $71.1^{*}(5.5)$ & $73.5^{*}, \#(5.4)$ & $76.0^{\#}(8)$ & $84.2(6.6)$ \\
IPT-LL $(\mathrm{Nm})$ & $76.1(7)$ & $86.6(7.2)$ & $91.3(8.3)$ & $90.9(10.1)$ \\
\hline
\end{tabular}

EPT: eccentric peak torque; CPT: concentric peak torque; IPT: isometric peak torque at $110^{\circ}$ knee joint ankle.

Data are reported as mean (standard error of the mean).

* Significantly different $(P<0.05)$ compared to baseline data.

\# Significantly different $(P<0.05)$ between the exercise bouts with SL and LL of rectus femoris. 
clinically applied in cases where there is a change in hip angle without significant changes in the knee angle.

The present findings on muscle damage contradict published data according to which greater muscle damage were observed following eccentric exercise with long compared to short muscle lengths (Jones et al., 1989; Newham et al., 1988). These authors proposed that, during eccentric exercise at long muscle length, actin and myosin are pulled so far apart that only a few or no cross-bridges are present in the sarcomere, which could then disrupt the adjacent sarcolemma or sarcoplasmic reticulum as a secondary event, leading to their damage.

Methodological variations may account for this discrepancy and the use of muscles at different anatomical sites may be one of them. For instance, since arm muscles - used in previous studies (Jones et al., 1989; Nosaka and Sakamoto, 2001) - are not gravitational, their recovery rate from injury might be different from that of leg muscles. Another variation may be the greater number of maximal voluntary eccentric actions used in this study. We introduced 120 MVCs whereas the only other study that addressed the same question used approximately half of this number (Child et al., 1998). However, the methodology of the present investigation does not allow us to fully understand the implications of an increased number of MVCs in relation to muscle damage. The way of setting short and long muscle length may be yet another methodological variation. Alterations of the knee active range of motion were previously obtained by changing the length of both rectus femoris and vasti muscles (Child et al., 1998). In contrast, the present active range of motion remained the same (i.e., $120^{\circ}$ ) for both eccentric exercise sessions and the altered hip angle only affected the biarticular rectus femoris and sartorious.

Our findings on muscle damage are further supported by the fact that the studied muscle performance revealed significantly greater declines after eccentric exercise with SL compared to LL of rectus femoris. The improved motor unit recruitment found during SL as opposed to LL exercise (Maffiuletti and Lepers, 2003) may partly explain the current results. Indeed, better motor unit recruitment is associated with greater torque outputs, which inevitably, bring the exercising muscle closer to its damage limit. Nevertheless, our findings on muscle performance contradict published data indicating greater decline after eccentric exercise with LL compared to SL (Jones et al., 1989; Nosaka and Sakamoto, 2001).

The CK activity was significantly elevated up to 96 hours post-exercise after the two modes of eccentric exercise. This significant elevation of CK was also observed in studies where the same mode of exercise was used to induce muscle damage (Child et al., 1998; Newham et al., 1988; Nosaka and Sakamoto, 2001). In the present study, however, only at $72 \mathrm{~h}$ post-exercise CK activity revealed significant greater elevation after SL eccentric exercise compared to LL equivalent. The lack of further significant $\mathrm{CK}$ differences between the two eccentric exercise modes could be attributed to the high inter-individual variability in the enzyme response, which has already been confirmed in subjects comparable to those participated in the present investigation (Newham et al., 1983) as well as in an animal study (Friden and Lieber, 2001).

The significant elevations of delayed onset muscle soreness found herein are in line with at least two sets of published data in which eccentric exercise was adopted to induce muscle damage (Jamurtas et al., 2000; Smith et al., 1994). In these two studies, as in the present one, the delayed onset muscle soreness of subjects elevated several times above baseline data in response to unaccustomed exercise.

Although the current muscle damage indicators have been previously used in studies of similar nature (Armstrong, 1990; Brown et al., 1999; Clarkson and Tremblay, 1988), limitations might arise from the their use. For instance, delayed onset muscle soreness and RoM are subjective measurements as they can be affected by the individuals' pain tolerance. The introduction of EMG could, perhaps, reduce the subjectiveness of these parameters.

In conclusion, and within the limitations of the present study, eccentric exercise at SL of rectus femoris induces greater muscle damage and causes greater muscle performance decline than the corresponding LL. Further work is required in this under-researched, but potentially important area, with an emphasis on assessing muscle length at the fascicle level.

\section{References}

Armstrong, R.B., 1990. Initial events in exercise-induced muscular injury. Med. Sci. Sports Exerc. 22, 429-435.

Babault, N., Pousson, M., Michaut, A., Van Hoecke, J., 2003. Effect of quadriceps femoris muscle length on neural activation during isometric and concentric contractions. J. Appl. Physiol. 94, 983990.

Brown, S., Day, S., Donnelly, A., 1999. Indirect evidence of human skeletal muscle damage and collagen breakdown after eccentric muscle actions. J. Sports Sci. 17, 397-402.

Child, R.B., Saxton, J.M., Donnelly, A.E., 1998. Comparison of eccentric knee extensor muscle actions at two muscle lengths on indices of damage and angle-specific force production in humans. J. Sports Sci. 16, 301-308.

Clarkson, P.M., Tremblay, I., 1988. Exercise-induced muscle damage, repair, and adaptation in humans. J. Appl. Physiol. 65, 1-6.

Delp, S.L., Loan, J.P., 1995. A graphics-based software system to develop and analyze models of musculoskeletal structures. Comput. Biol. Med. 25, 21-34.

Friden, J., Lieber, R.L., 2001. Eccentric exercise-induced injuries to contractile and cytoskeletal muscle fibre components. Acta Physiol. Scand. 171, 321-326.

Hoppeler, H., 1986. Exercise-induced ultrastructural changes in skeletal muscle. Int. J. Sports Med. 7, 187-204. 
Hunter, K.D., Faulkner, J.A., 1997. Plyometric contraction-induced injury of mouse skeletal muscle: effect of initial length. J. Appl. Physiol. 82, 278-283.

Jamurtas, A.Z., Fatouros, I.G., Buckenmeyer, P., Kokkinidis, E., Taxildaris, K., Kambas, A., Kyriazis, G., 2000. Effects of plyometric exercise on muscle damage and plasma creatine kinase levels and its comparison with eccentric and concentric exercise. J. Strength Cond. Res. 14, 68-74.

Jones, D.A., Newham, D.J., Torgan, C., 1989. Mechanical influences on long-lasting human muscle fatigue and delayed-onset pain. J. Physiol. 412, 415-427.

Kay, D., St. Clair Gibson, A., Mitchell, M.J., Lambert, M.I., Noakes, T.D., 2000. Different neuromuscular recruitment patterns during eccentric, concentric and isometric contractions. J. Electromyogr. Kinesiol. 10, 425-431.

Komi, P.V., Bosco, C., 1978. Utilization of stored elastic energy in leg extensor muscles by men and women. Med. Sci. Sports. 10, 261265 .

Koutedakis, Y., Frischknecht, R., Murthy, M., 1997. Knee flexion to extension peak torque ratios and low-back injuries in highly active individuals. Int. J. Sports Med. 18, 290-295.

MacIntyre, D.L., Slawnych, M.P., Reid, W.D., McKenzie, D.C., 1998. Fatigue of the knee extensor muscles following eccentric exercise. Electromyogr. Clin. Neurophysiol. 38, 3-9.

Macpherson, P.C., Dennis, R.G., Faulkner, J.A., 1997. Sarcomere dynamics and contraction-induced injury to maximally activated single muscle fibres from soleus muscles of rats. J. Physiol. $500(\mathrm{Pt}$ 2), 523-533.

Maffiuletti, N.A., Lepers, R., 2003. Quadriceps femoris torque and EMG activity in seated versus supine position. Med. Sci. Sports Exerc. 35, 1511-1516.

McHugh, M.P., Tetro, D.T., 2003. Changes in the relationship between joint angle and torque production associated with the repeated bout effect. J. Sports Sci. 21, 927-932.

McNair, P., Marshall, R., Matheson, J., 1991. Quadriceps strength deficit associated with rectus femoris rupture: a case report. Clin. Biomech. 6, 190-192.

Morgan, D.L., 1990. New insights into the behavior of muscle during active lengthening. Biophys. J. 57, 209-221.
Newham, D.J., Jones, D.A., Clarkson, P.M., 1987. Repeated highforce eccentric exercise: effects on muscle pain and damage. J. Appl. Physiol. 63, 1381-1386.

Newham, D.J., Jones, D.A., Edwards, R.H., 1983. Large delayed plasma creatine kinase changes after stepping exercise. Muscle Nerve 6, 380-385.

Newham, D.J., Jones, D.A., Ghosh, G., Aurora, P., 1988. Muscle fatigue and pain after eccentric contractions at long and short length. Clin. Sci. (Lond.) 74, 553-557.

Nosaka, K., Newton, M., Sacco, P., 2002. Delayed-onset muscle soreness does not reflect the magnitude of eccentric exerciseinduced muscle damage. Scand. J. Med. Sci. Sports 12, 337-346.

Nosaka, K., Sakamoto, K., 2001. Effect of elbow joint angle on the magnitude of muscle damage to the elbow flexors. Med. Sci. Sports Exerc. 33, 22-29.

Paddon-Jones, D., Abernethy, P.J., 2001. Acute adaptation to low volume eccentric exercise. Med. Sci. Sports Exerc. 33, 1213-1219.

Pavol, M.J., Grabiner, M.D., 2000. Knee strength variability between individuals across ranges of motion and hip angles. Med. Sci. Sports Exerc. 32, 985-992.

Prou, E., Guevel, A., Benezet, P., Marini, J.F., 1999. Exercise-induced muscle damage: absence of adaptive effect after a single session of eccentric isokinetic heavy resistance exercise. J. Sports. Med. Phys. Fitness 39, 226-232.

Saxton, J.M., Clarkson, P.M., James, R., Miles, M., Westerfer, M., Clark, S., Donnelly, A.E., 1995. Neuromuscular dysfunction following eccentric exercise. Med. Sci. Sports Exerc. 27, 1185-1193.

Sayers, S.P., Clarkson, P.M., 2001. Force recovery after eccentric exercise in males and females. Eur. J. Appl. Physiol. 84, 122-126.

Smith, L.L., Fulmer, M.G., Holbert, D., McCammon, M.R., Houmard, J.A., Frazer, D.D., Nsien, E., Israel, R.G., 1994. The impact of a repeated bout of eccentric exercise on muscular strength, muscle soreness and creatine kinase. Br. J. Sports Med. 28, 267-271.

Thomas, J., Nelson, J., 1996. Research methods in physical activity, Human Kinetics, Champaing.

Vollestad, N.K., Sejersted, O.M., Bahr, R., Woods, J.J., BiglandRitchie, B., 1988. Motor drive and metabolic responses during repeated submaximal contractions in humans. J. Appl. Physiol. 64, $1421-1427$. 